\section{Turbulente Zeiten in der Rheumatologie}

W ir schreiben das Jahr 1997: Die Lebenserwartung von Patienten mit rheumatischen Erkrankungen ist gegenüber Gesunden um zehn Jahre verkürzt, etwa die Hälfte der Patienten sind nach fünf Krankheitsjahren vollinvalidisiert, mehrere Gelenkoperationen pro Jahr sind die Regel. In den Wartezimmern der Rheumatologen sind Gehhilfen und Rollstühle der wartenden Patienten ein vertrauter Anblick.

Ein Sprung ins Jahr 2017: Therapieziel ist es heute, dass der Patient mit rheumatoider Arthritis (RA) nichts mehr von seiner Erkrankung spürt und seine volle Arbeitsfähigkeit und Lebensqualität behält. Röntgenkontrollen verlieren an Bedeutung, weil auf den Bildern meist kaum noch etwas von der RA zu sehen ist. Funktionsstatus und Lebenserwartung nähern sich den aus der Normalbevölkerung bekannten Zahlen.

Wie ist dieser dramatische Wandel in nur zwanzig Jahren erklärbar? Zum einen durch Fortschritte bei der Aufklärung der Pathogenese, zum anderen wurden die Früherkennung der RA wie auch der Spondyloarthritiden verbessert. Zudem gibt es inzwischen ausgezeichnete Leitlinien, an denen sich der Arzt orientieren kann, und es haben sich erfolgreiche Therapiestrategien herauskristallisiert. Einen entscheidenden Anteil an den Fortschritten hat aber die Entwicklung und Etablierung der hochwirksamen und ausgezeichnet verträglichen Biologika.

Ausgehend von den ersten Biologika Infliximab und Etanercept verfügen wir mittlerweile in der Rheumatologie über 13 verschiedene Wirkstoffe, welche unter anderem auch in der Kinder-Rheumatologie die Behandlungsmöglichkeiten dramatisch verbessert haben. Bekanntermaßen handelt es sich um sehr kostenintensive Substanzen. Allerdings ist diese Kostenstruktur mit der Einführung von Rabattverträgen und Biosimilars in Bewegung geraten. Davon abgesehen führen die therapeutischen Fortschritte zu beträchtlichen Einsparungen: deutlich weniger Klinikaufenthalte und Operationen bei Rheuma-Patienten, viel weniger Arbeitsausfallstage. Darüber wird seltsamerweise viel weniger

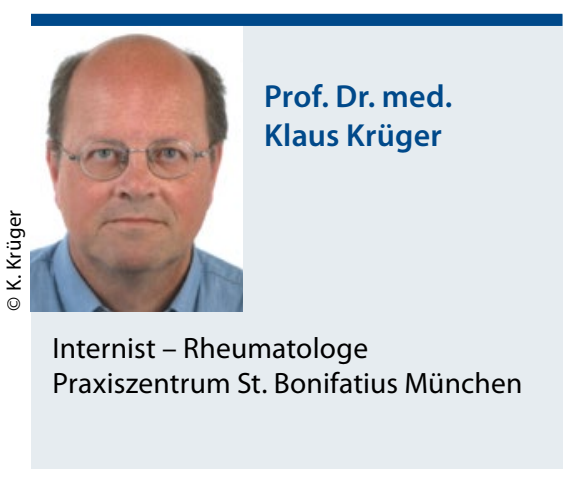

geredet wird als über die Medikamentenkosten.

Also, Rundumzufriedenheit in der Rheumatologie? Leider nicht ganz. Die Versorgungssituation für die deutschen RA-Patienten hat sich in den vergangenen Jahrzehnten zwar verbessert, aber bei Weitem nicht genug. Nach wie vor gibt es nur ein Drittel der benötigten Rheumatologen. Ein großer Fortschritt ist die Etablierung der rheumatologischen Fachassistentin, die seit 2006 in den Praxen den Rheumatologen in vielerlei Hinsicht entlasten kann. Damit allein wird das Problem der Minderversorgung allerdings nicht zu lösen sein.

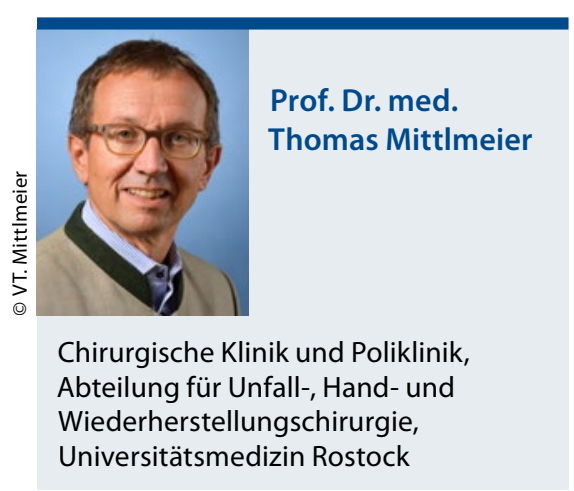

BV bietet somit die Möglichkeit, neben der Syndesmose auch die anatomisch korrekte Reposition des hinteren Kantendreiecks zu beurteilen. Die unter Nutzung des 3-D-BV beinahe konstante Rate von mehr als $20 \%$ intraoperativer Revisionen auch in der Hand des Erfahrenen, unterstreicht den klinischen Nutzen dieses Verfahrens, das eine postoperative CT und einen eventuellen Sekundäreingriff entbehrlich macht. 